\title{
GenoType MTBDR assays for the diagnosis of multidrug-resistant tuberculosis:
} a meta-analysis

\author{
D.I. Ling* ${ }^{*}$ A.A. Zwerling* and M. Pai,*\#
}

ABSTRACT: The global extensively drug-resistant tuberculosis (TB) response plan calls for implementation of rapid tests to screen patients at risk of drug-resistant TB. Currently, two line probe assays exist, the INNO-LiPA ${ }_{\triangleright}$ Rif.TB assay (Innogenetics, Ghent, Belgium) and the GenoType $_{\circledR}$ MTBDR assay (Hain LifeScience $\mathrm{GmbH}$, Nehren, Germany). While LiPA studies have been reviewed, the accuracy of GenoType assays has not been systematically reviewed.

The present authors carried out a systematic review and used meta-analysis methods appropriate for diagnostic accuracy. After the literature searches, 14 comparisons for rifampicin and 15 comparisons for isoniazid were identified in 10 articles that used GenoType MTBDR assays. Accuracy results were summarised in forest plots and pooled using bivariate randomeffects regression.

The pooled sensitivity $(98.1 \%$, $95 \%$ confidence interval $(\mathrm{Cl})$ 95.9-99.1) and specificity $(98.7 \%$, 95\% Cl 97.3-99.4) estimates for rifampicin resistance were very high and consistent across all subgroups, assay versions and specimen types. The accuracy for isoniazid was variable, with lower sensitivity $(84.3 \%, 95 \% \mathrm{Cl} 76.6-89.8)$ and more inconsistent than specificity $(99.5 \%, 95 \% \mathrm{Cl}$ 97.5-99.9).

GenoType MDTBR assays demonstrate excellent accuracy for rifampicin resistance, even when used on clinical specimens. While specificity is excellent for isoniazid, sensitivity estimates were modest and variable. Together with data from demonstration projects, the meta-analysis provides evidence for policy making and clinical practice.

KEYWORDS: Diagnostic accuracy, drug resistance, line probe assay, multidrug-resistant tuberculosis, sensitivity and specificity, tuberculosis

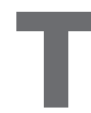
uberculosis (TB) is a major global health problem [1]. The emergence of multidrugresistant (MDR)-TB and, more recently, of extensively drug-resistant (XDR)-TB, are widely considered to be serious threats to global TB control [2-4]. Conventional drug-susceptibility testing (DST) has limitations. Solid media-based techniques, such as Löwenstein-Jensen and Middlebrook 7H10/11 using the proportion, absolute concentration and resistance ratio methods, take up to 8-12 weeks [5]. Liquid media-based methods, such as the BACTEC ${ }_{\mathbb{B}}$ (BD Diagnostics, Sparks, MD, USA), MGIT® (BD Diagnostics) and BacT/ALERT ${ }_{\circledR}$ (bioMérieux SA, Marcy l'Etoile, France) systems, are faster and sensitive, but more expensive and complex [6].

The World Health Organization (WHO) and partners have proposed a global XDR-TB response plan, which calls for wide-scale implementation of rapid methods to screen patients at risk of MDR-TB [7]. Rapid tests can provide results within days (even without culture, i.e. directly on specimens) and thus enable prompt and appropriate treatment, decrease morbidity and mortality, and interrupt transmission. Line probe assays (based on reverse-hybridisation DNA strip technology) could potentially address this urgent need [5].

Currently, two commercial line probe assays exist, the INNO-LiPA ${ }$ Rif.TB (Innogenetics, Ghent, Belgium) and GenoType ${ }_{\circledR}$ MTBDR (Hain LifeScience GmbH, Nehren, Germany). The LiPA test can simultaneously detect Mycobacterium tuberculosis and the presence of a mutation in the $r p o B$ gene, which confers resistance to rifampicin [8]. A recent meta-analysis summarised the results obtained for the INNO-LiPA Rif.TB test and showed that LiPA had high sensitivity and specificity when Mycobacterium tuberculosis isolates from culture were used. The
AFFILIATIONS

*Dept of Epidemiology, Biostatistics and Occupational Health, McGill University, and

\#Respiratory Epidemiology and Clinical Research Unit, Montreal Chest Institute, Montreal, QC, Canada.

CORRESPONDENCE

M. Pai

Dept of Epidemiology, Biostatistics and Occupational Health

McGill University

1020 Pine Avenue West

Montreal

QC H3A 1 A2

Canada

Fax: 15143984503

E-mail: madhukar.pai@mcgill.ca

Received

April 232008

Accepted after revision:

June 272008

\section{SUPPORT STATEMENT}

The present study was supported by funding from the World Health Organization, Geneva, Switzerland.

\section{STATEMENT OF INTEREST}

A statement of interest for M. Pai can be found at www.erj.ersjournals.com/ misc/statements.shtm 
majority of studies had sensitivity of $\geqslant 95 \%$, and nearly all were $100 \%$ specific [9]. The results, however, were less accurate when the test was directly applied on clinical specimens (i.e. sputum), and fewer data were available [9].

The GenoType MTBDR assay, introduced in 2004, includes three steps: DNA extraction, multiplex PCR amplification, and reverse hybridisation [10]. The MTBDR assay has an additional advantage over the LiPA because it can detect both rifampicin and isoniazid resistance. The MTBDR assay identifies mutations in the $r p o B$ gene as well as mutations in the kat $G$ gene for high-level isoniazid resistance [10]. The MTBDRplus, the second-generation assay, also detects mutations in the inh $A$ gene that confers resistance to low-levels of isoniazid [10]. A systematic review and meta-analysis was performed to determine the accuracy of GenoType MTBDR assays for diagnosing MDR-TB in clinical specimens and culture isolates. In addition to estimating the overall accuracy, the quality of studies was evaluated and factors were explored that may be responsible for heterogeneity among studies.

\section{METHODS}

To perform the current meta-analysis, standard methods were used that are appropriate for systematic reviews of diagnostic accuracy studies [11-13].

\section{Search strategy}

The present authors systematically searched the literature using predetermined inclusion criteria: use of either the first or second-generation GenoType MTBDR assay for diagnosing drug-resistant $\mathrm{TB}$, comparison of the assay result with conventional DST as a reference standard, information to calculate sensitivity and specificity and a minimum sample size of 20 to avoid potential selection bias in small studies [14].

Three databases (PubMed, EMBASE and BIOSIS) were searched for relevant English language citations. Search terms included "tuberculosis", "Mycobacterium tuberculosis", "Hain LifeScience", "line probe assay", "GenoType MTBDR" and "molecular diagnostic techniques". The search was restricted to the time period January 2004 to March 2008, since the first generation MTBDR assay was introduced in October 2004, and the second-generation MTBDRplus assay became available in February 2007. Reference lists from included studies were also searched. In addition, laboratory experts and the test manufacturer (Hain LifeScience $\mathrm{GmbH}$ ) were contacted for additional studies. Conference abstracts were included when sufficient data were reported.

\section{Study selection}

Figure 1 shows the study selection process. After screening titles and abstracts, 22 articles were eligible for full text review after excluding articles that were not TB-related, did not focus on MDR-TB or used other line probe assays. Of these, 12 articles that used other GenoType assays or did not contain enough information to calculate sensitivity and specificity were further excluded from data extraction, and ten studies (including one conference abstract [15]) that used MTBDR assays for the detection of drug resistance were included in the present meta-analysis [15-24]. Several studies made more than one comparison, in which case each comparison was considered separately. Thus, the total number of comparisons in

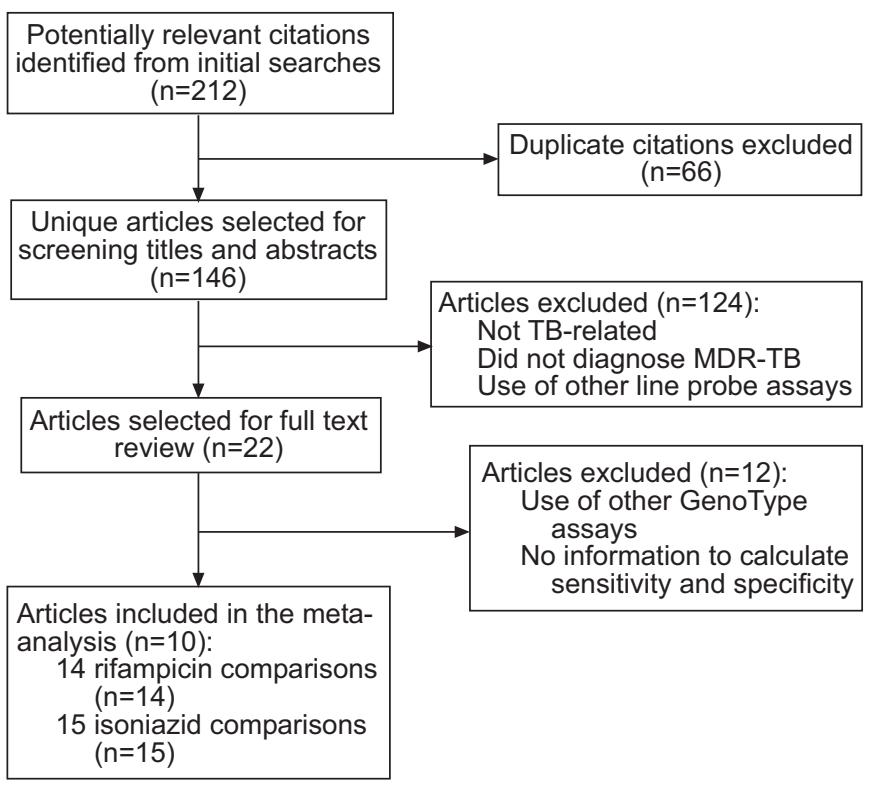

FIGURE 1. Study selection process. TB: tuberculosis; MDR: mutidrugresistant. GenoType MTBDR is manufactured by Hain LifeScience GmbH (Nehren, Germany).

the final analysis was 14 for detection of rifampicin resistance and 15 for detection of isoniazid resistance.

\section{Data extraction}

A data extraction form was created and piloted with a subset of eligible studies. Based on the experience gained in the pilot data extraction, the data extraction form was improved and finalised. The final set of studies was assessed with the standardised form by one reviewer (D.I. Ling) and crosschecked by a second (A.A. Zwerling). Any differences between reviewers were resolved by consensus. Since discrepant analysis (where discordant results between line probe assay and reference standard results are resolved, post-hoc, using clinical or other test data) may be a potential source of bias, unresolved data were preferentially included when available [25].

\section{Assessment of study quality}

Using the Quality Assessment of Studies of Diagnostic Accuracy included in Systematic Reviews (QUADAS) criteria [26], the quality characteristics that are important for diagnostic accuracy studies were assessed: 1) comparison of the index test with an appropriate reference standard; 2) blinded interpretation of the test result with reference standard results and vice versa; 3 ) complete verification of test results with the reference standard; 4) recruitment of patients either consecutively or randomly; and 5) study design (i.e. cross-sectional versus case-control design).

\section{Meta-analysis methods}

Sensitivity and specificity values were calculated for the GenoType tests investigated in each study, along with their $95 \%$ confidence intervals, and displayed as forest plots. Sensitivity is the proportion of resistant results that are 


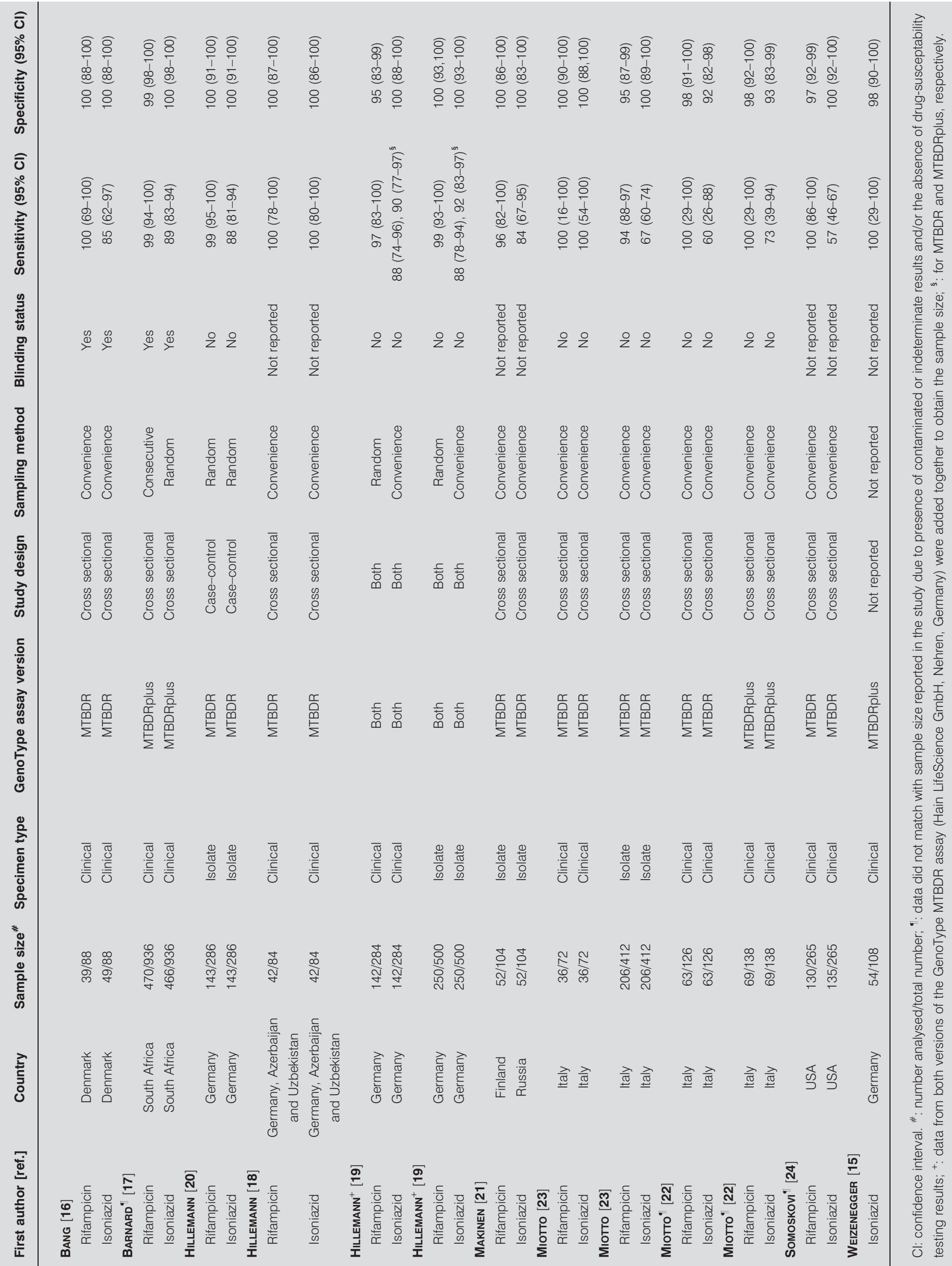


correctly classified by the MTBDR assay, while specificity is the proportion of susceptible results that are correctly identified as susceptible by the commercial kit.

Each study in the meta-analysis contributed a pair of numbers: sensitivity (true-positivity rate) and specificity (one minus false-positivity rate). Since these measures tend to be correlated and vary with the thresholds (cut-off points) used across individual studies, a summary receiver operating characteristic (SROC) curve analysis was performed in order to explore the effect of thresholds on the results $[11,27]$. The SROC curve displays the sensitivity and specificity estimates from each study within the receiver operating characteristic space. A regression curve is fitted through the distribution of pairs of sensitivity and specificity. A shoulder-like curve indicates that heterogeneity between studies may be due to the threshold effect (i.e. variation in cut-offs across studies) and that a common diagnostic odds ratio (DOR) exists that does not change with the threshold [27-29]. A nonshoulder-like curve shows that sensitivity and specificity are not correlated. In addition, the area under the curve (AUC) also estimates the overall diagnostic accuracy. An AUC of 50\% would indicate poor discriminatory ability, while an AUC of $100 \%$ means that the test discriminates perfectly between resistant and susceptible strains [27-29].

In addition to the SROC analyses, bivariate random effects regression analyses [13] were performed in STATA/IC 10.0 (Stata Corporation, Texas, USA, 2007) using the program "metandi" [30] to generate pooled accuracy estimates of sensitivity, specificity, positive likelihood ratio (LR+), negative likelihood ratio (LR-), and DOR. The LR+ measures how much more frequent a positive result (i.e. resistant) is found in

\section{TABLE 2 Study characteristics}

\begin{tabular}{ll} 
Characteristic & Frequency $^{\#}$ \\
\hline Assay version & \\
MTBDR & 6 \\
MTBDRplus & 2 \\
Both & 2 \\
Sample tested & \\
Clinical specimen & 6 \\
Culture isolate & 2 \\
Both & 2 \\
Conventional DST method used & \\
Agar proportion & 1 \\
BACTEC 460 & 3 \\
BACTEC MGIT 960 & 3 \\
BACTEC 460 \& MGIT 960 & 2 \\
Not reported & 1 \\
Data & \\
Resolved & 2 \\
Not resolved &
\end{tabular}

MTBDR and MTBDRplus are manufactured by Hain LifeScience $\mathrm{GmbH}$ (Nehren, Germany). BACTEC 460 and MGIT 960 are manufactured by BD Diagnostics (Sparks, MD, USA). DST: drug-susceptability testing; ${ }^{*}: n=10$ studies; ${ }^{\circ}$ : after discrepant analysis; ${ }^{+}$: discrepant analysis not performed. resistant versus susceptible strains. Conversely, the LRmeasures how much more likely a negative result (i.e. susceptible) is found in resistant versus susceptible strains. The DOR, or the odds of a positive result in resistant strains compared with the odds of a positive result in susceptible strains, combines both likelihood ratios and is a global measure of test performance [31]. The DOR is calculated by LR+/LR- or (sensitivity/(1-specificity))/((1-sensitivity)/specificity) [31].

As described by REITSMA et al. [13], the bivariate regression method assumes that the sensitivity values from individual studies (after logit transformation) within a meta-analysis are approximately normally distributed around a mean value with a certain amount of variability around this mean [13]. This is a random effects approach. This variation in underlying sensitivity estimates between studies can be related to undetected differences in study population, differences in implicit threshold (cut-off), or unnoticed variations in the index test protocol. These considerations also apply to specificity estimates. The potential presence of a (negative) correlation between sensitivity and specificity within studies is addressed by explicitly incorporating this correlation into the analysis. The combination of two normally distributed outcomes, the logit-transformed sensitivity and specificity values, while acknowledging the possible correlation between them, leads to the bivariate normal distribution [13]. The bivariate approach overcomes the problems associated with simple pooling (i.e. weighted average) of sensitivity and specificity estimates $[12,13]$.

\section{Subgroup analysis}

Heterogeneity is usually a concern with meta-analyses and refers to a high degree of variability in accuracy estimates across studies [32]. Heterogeneity could be due to variability in thresholds, prevalence of drug-resistance, populations studied, variations in assay methods and reference standard tests and

TABLE 3 Assessment of study quality

\begin{tabular}{|c|c|}
\hline Characteristic & Frequency ${ }^{\#}$ \\
\hline \multicolumn{2}{|l|}{ Study design } \\
\hline Cross sectional & 7 \\
\hline Case-control & 1 \\
\hline Both & 1 \\
\hline Not reported & 1 \\
\hline \multicolumn{2}{|c|}{ Recruitment (sampling) method } \\
\hline Random sampling & 1 \\
\hline Consecutive and random & 1 \\
\hline Convenience sampling & 6 \\
\hline Convenience and random & 1 \\
\hline Not reported & 1 \\
\hline \multicolumn{2}{|l|}{ Verification } \\
\hline Complete & 10 \\
\hline \multicolumn{2}{|l|}{ Blinded interpretation } \\
\hline Yes & 2 \\
\hline No & 4 \\
\hline Not reported & 4 \\
\hline
\end{tabular}



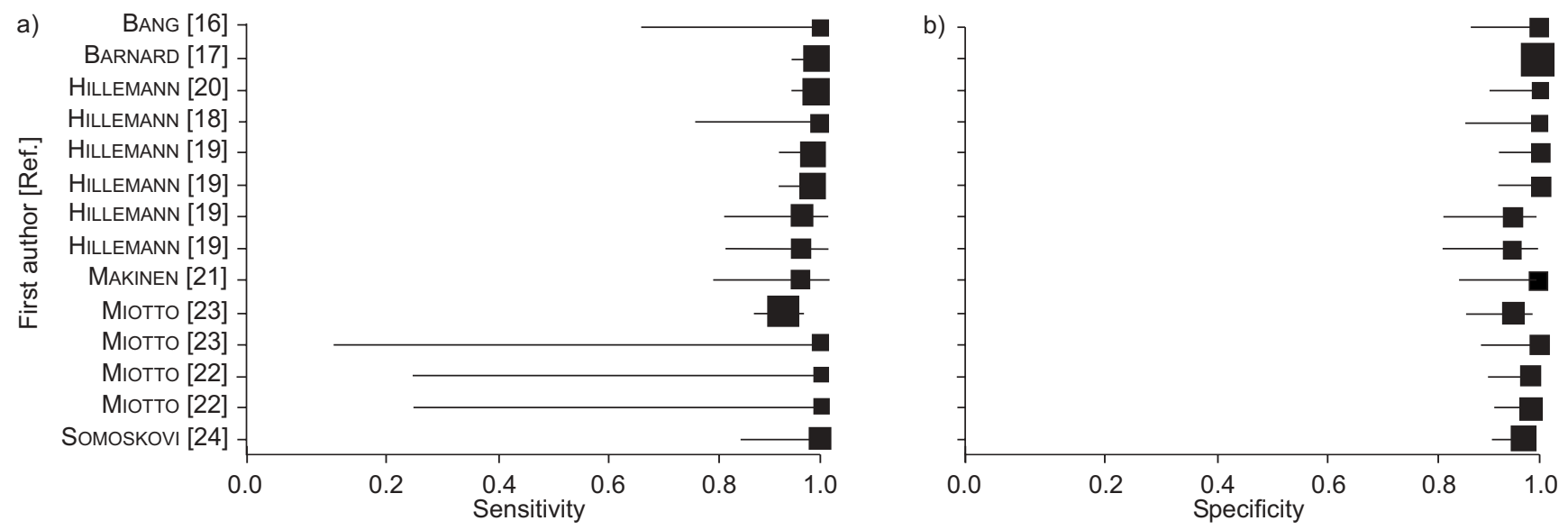

FIGURE 2. Forest plot of sensitivity (a) and specificity (b) estimates for rifampicin resistance (all 14 studies, regardless of specimen type or assay version). point estimates of sensitivity and specificity from each study (proportionate to size of the study); : $95 \%$ confidence intervals. See also table 4.

\section{TABLE 4 Sensitivity and specificity estimates for rifampicin}

\begin{tabular}{|c|c|c|}
\hline First author [ref.] & Sensitivity ${ }^{\#}$ & Specificity \\
\hline BANG [16] & $1.00(0.69-1.00)$ & $1.00(0.88-1.00)$ \\
\hline BARNARD [17] & $0.99(0.94-1.00)$ & $0.99(0.98-1.00)$ \\
\hline HiLLEmann [20] & $0.99(0.95-1.00)$ & $1.00(0.91-1.00)$ \\
\hline HiLLemanN [18] & $1.00(0.78-1.00)$ & $1.00(0.87-1.00)$ \\
\hline HiLLemanN [19] & $0.99(0.93-1.00)$ & $1.00(0.93-1.00)$ \\
\hline HillemanN [19] & $0.99(0.93-1.00)$ & $1.00(0.93-1.00)$ \\
\hline HillemanN [19] & $0.97(0.83-1.00)$ & $0.95(0.83-0.99)$ \\
\hline HiLLemanN [19] & $0.97(0.83-1.00)$ & $0.95(0.83-0.99)$ \\
\hline MAKINEN [21] & $0.96(0.82-1.00)$ & $1.00(0.86-1.00)$ \\
\hline Мıотто [23] & $0.94(0.88-0.97)$ & $0.95(0.87-0.99)$ \\
\hline Мıтто [23] & $1.00(0.16-1.00)$ & $1.00(0.90-1.00)$ \\
\hline Мıтто [22] & $1.00(0.29-1.00)$ & $0.98(0.91-1.00)$ \\
\hline Мıтто [22] & $1.00(0.29-1.00)$ & $0.98(0.92-1.00)$ \\
\hline Soмоsкоvı [24] & $1.00(0.86-1.00)$ & $0.97(0.92-0.99)$ \\
\hline \multicolumn{3}{|c|}{$\begin{array}{l}\text { Data are presented in \% (confidence interval). }{ }^{\#}: \text { Chi-squared }=12.57 \text {; degrees } \\
\text { of freedom }=13(p=0.4817) ; \text { inconsistency }(I-\text { squared })=0.0 \% ;{ }^{*} \text { : Chi-squared }= \\
\text { 18.75; degrees of freedom }=13(p=0.1310) \text {; inconsistency }(I-\text { squared })=30.7 \% \\
\text { See also figure } 2 \text {. }\end{array}$} \\
\hline
\end{tabular}

differences in study quality. When significant heterogeneity is present, summary estimates from meta-analyses are not meaningful. Heterogeneity was detected using Chi-squared and I-squared tests [33]. Further reasons for the heterogeneity were investigated by pre-specified subgroup (stratified) analysis [32]. In the subgroup analysis, the data were stratified according to the type of sample tested (clinical specimen versus culture isolate) and test version used (MTBDR versus MTBDRplus) to determine if accuracy varied across subgroups. Accuracy for rifampicin resistance was estimated separately from accuracy for isoniazid resistance.

\section{RESULTS}

\section{Characteristics of included studies}

As shown in figure 1, 10 articles were included, with 14 comparisons for the detection of rifampicin resistance and 15 comparisons for the detection of isoniazid resistance, for a total of 3,349 specimens (mean (range) 116 (36-470)). Tables 1-3 show the characteristics of the 10 studies in the meta-analysis. More studies assessed the performance of the MTBDR assay, because the MTBDRplus has only been available since 2007. The majority of the studies detected drug resistance on clinical specimens, including both smear-positive and smear-negative sputum, other respiratory samples and nonrespiratory samples. In addition, most laboratories used the BACTEC 460 or MGIT 960 (both from BD Diagnostics) systems for conventional DST.

TABLE 5 Pooled summary estimates for rifampicin resistance

\begin{tabular}{lccccc} 
Subgroup & Pooled sensitivity & Pooled specificity & Pooled LR+ & Pooled LR- & Pooled DOR \\
\hline All rifampicin studies\# $^{*}$ & $98.1(95.9-99.1)$ & $98.7(97.3-99.4)$ & $78.0(36.3-168.0)$ & $0.02(0.01-0.04)$ & $4010.1(1205.9-13335.2)$ \\
Only MTBDRplus assays $^{*}$ & $98.4(95.1-99.5)$ & $98.9(96.8-99.7)$ & $95.3(30.7-296.0)$ & $0.02(0.005-0.05)$ & $6150.7(1061.8-35628.9)$ \\
Only clinical specimens $^{+}$ & $98.6(95.5-99.6)$ & $98.5(96.9-99.3)$ & $66.3(31.9-138.0)$ & $0.01(0.004-0.04)$ & $4659.3(1064.6-20391.3)$ \\
\hline
\end{tabular}

Data are presented as \% (confidence interval). MTBDRplus is manufactured by Hain LifeScience GmbH (Nehren, Germany). LR+: positive likelihood ratio; LR-: negative likelihood ratio; DOR: diagnostic odds ratio. ${ }^{\#}$ : number of comparisons was $14 ;{ }^{\prime}$ : number of comparisons was 4 ; $^{+}$: number of comparisons was 9. 


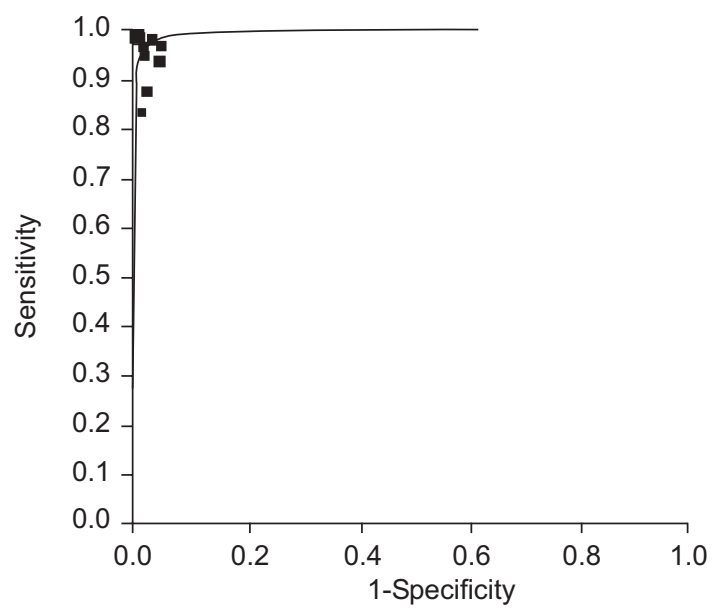

FIGURE 3. Summary reciever operating characteristic (SROC) plot for rifampicin resistance (all 14 studies, regardless of specimen type or assay version). - : each study in the meta-analysis size proportional to size of study; regression line that summarises the overall diagnostic accuracy. Area under the curve (AUC) 0.9949; SE of AUC 0.0023; point of the SROC curve where the sensitivity and specificity are equal $\left(Q^{*}\right) 0.9722$; $S E$ of $Q^{*} 0.0073$.

Unresolved data, in which discrepant analysis was not performed, were available in eight out of the 10 studies.

Most of the studies were cross-sectional in design (table 3). Only two studies used consecutive or random sampling, while seven studies recruited patients using convenience sampling methods. One review of 31 meta-analyses on several diseases found higher accuracy measures associated with studies that used nonconsecutive sampling methods [34]. All studies reported complete verification of MTBDR results with conventional DST as the reference standard. Past evidence has shown that investigators do not report all the study components in their publications $[34,35]$. In the present analysis, four studies did not report blinded interpretation of either the MTBDR or conventional DST results. Not blinding investigators to reference standard results when interpreting index test results has been shown to overestimate accuracy [36]. In
TABLE 6 Sensitivity and specificity estimates for isoniazid

\begin{tabular}{|c|c|c|}
\hline First author [ref.] & Sensitivity ${ }^{\#}$ & Specificity \\
\hline BANG [16] & $0.85(0.62-0.97)$ & $1.00(0.88-1.00)$ \\
\hline BaRnARD [17] & $0.89(0.83-0.94)$ & $1.00(0.98-1.00)$ \\
\hline Hillemann [20] & $0.88(0.81-0.94)$ & $1.00(0.91-1.00)$ \\
\hline Hillemann [18] & $1.00(0.80-1.00)$ & $1.00(0.86-1.00)$ \\
\hline HiLLEMANN [19] & $0.88(0.78-0.94)$ & $1.00(0.93-1.00)$ \\
\hline Hillemann [19] & $0.92(0.83-0.97)$ & $1.00(0.93-1.00)$ \\
\hline HiLLEMANN [19] & $0.88(0.74-0.96)$ & $1.00(0.88-1.00)$ \\
\hline HiLLemanN [19] & $0.90(0.77-0.97)$ & $1.00(0.88-1.00)$ \\
\hline WEIZENEGGER [15] & $1.00(0.29-1.00)$ & $0.98(0.90-1.00)$ \\
\hline MAKINEN [21] & $0.84(0.67-0.95)$ & $1.00(0.83-1.00)$ \\
\hline Мıотто [23] & $0.67(0.60-0.74)$ & $1.00(0.89-1.00)$ \\
\hline Мıотто [23] & $1.00(0.54-1.00)$ & $1.00(0.88-1.00)$ \\
\hline Мıтто [22] & $0.60(0.26-0.88)$ & $0.92(0.82-0.98)$ \\
\hline Мıотто [22] & $0.73(0.39-0.94)$ & $0.93(0.83-0.98)$ \\
\hline Soмosкovı [24] & $0.57(0.46-0.67)$ & $1.00(0.92-1.00)$ \\
\hline
\end{tabular}

Data are presented as \% (confidence interval). ${ }^{*}$ : Chi-squared $=84.93$; degrees of freedom=14 $(p<0.001)$; inconsistency $(I-$ squared $)=83.5 \%$; ${ }^{\text {: }}$ : Chi-squared $=$ 28.50; degrees of freedom $=14(p=0.0122)$; inconsistency $($ I-squared $)=50.9 \%$. See also figure 4

addition, most studies did not report whether patients were pre-treatment cases or already on treatment.

\section{Accuracy for rifampicin resistance}

Figure 2 and table 4 show the accuracy measures from all the rifampicin comparisons in a forest plot. Both sensitivity and specificity were highly consistent across the studies. Pooled sensitivity and specificity for rifampicin resistance was very high at $98.1 \%$ and $98.7 \%$, respectively. The Chi-squared and Isquared tests for heterogeneity in the summary results suggested no significant heterogeneity across studies. Thus, summary measures of the test's diagnostic ability for rifampicin resistance can adequately describe the data. Table 5 shows the pooled accuracy measures using the bivariate random
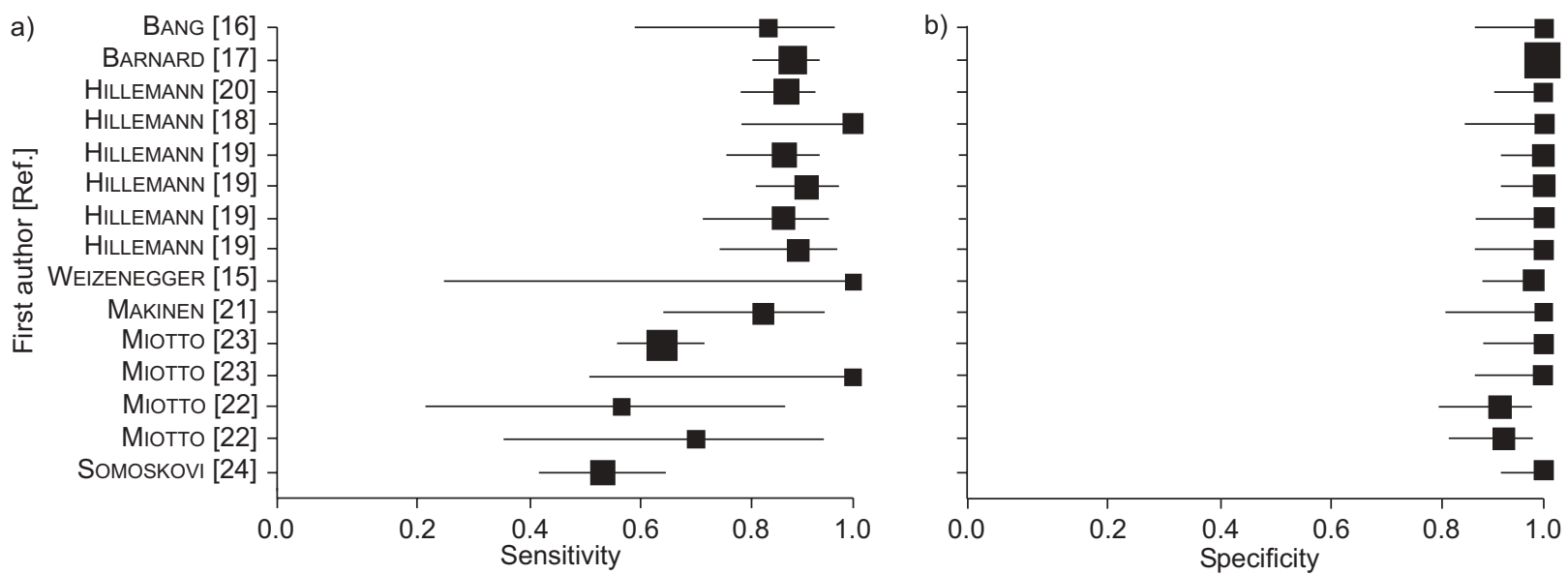

FIGURE 4. Forest plot of a) sensitivity and b) specificity estimates for isoniazid resistance (all 15 studies, regardless of specimen type or assay version). : point estimates of sensitivity and specificity from each study (proportional to size of the study). __ : 95\% confidence intervals. See also table 6. 


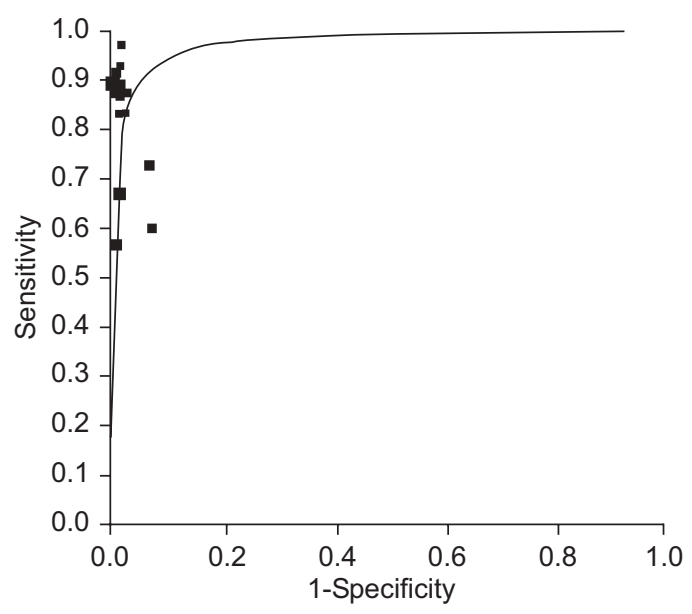

FIGURE 5. Summary reciever operating characteristic (SROC) plot for isoniazid resistance (all 15 studies, regardless of specimen type or assay version). 口: each study in the meta-analysis size proportional to size of study; regression line that summarises the overall diagnostic accuracy. Area under the curve (AUC) 0.9727; SE of AUC 0.0282; point on the SROC curve where the sensitivity and specificity are equal $\left(Q^{*}\right) 0.9243$; SE of $Q^{*} 0.0473$.

effects regression method. As seen in table 5, pooled sensitivity and specificity for rifampicin resistance was very high, and this was also reflected in the pooled likelihood ratios and DOR. The high accuracy is confirmed by the SROC plot shown in figure 3. The area under the SROC curve was 99\%, indicating near perfect discriminatory ability. Although the overall accuracy was consistently high across studies, subgroup analysis was performed by assay version and by specimen type. The accuracy was consistently high across all subgroups, specimen types and assay versions (table 5).

\section{Accuracy for isoniazid resistance}

Figure 4 and table 6 show the accuracy measures from all the isoniazid comparisons in a forest plot. Sensitivity estimates were highly heterogeneous across studies. In contrast, specificity estimates were fairly consistent across the studies. The tests for heterogeneity suggested significant variability across studies for sensitivity. The pooled sensitivity estimate should therefore be interpreted with caution. Figure 5 shows the SROC plot for isoniazid studies. The plot shows high specificity for most studies, but variable and modest sensitivity, ranging $57-100 \%$.
Overall, analyses of isoniazid studies clearly showed a high degree of variability in sensitivity estimates. This heterogeneity may result from differences in test methods, population and study characteristics [27]. Thus, subgroup analysis was performed to stratify data into relatively more homogeneous strata [32]. The current authors stratified the studies by test version as well as by sample type. As shown in table 7 , specificity did not vary across subgroups, but sensitivity was higher when only MTBDRplus studies were pooled. Specimen type did not seem to affect accuracy.

\section{DISCUSSION \\ Principal findings}

In the meta-analysis, the comprehensive literature search identified 14 comparisons for rifampicin resistance and 15 comparisons for isoniazid resistance in 10 articles that reported the use of MTBDR assays. Sensitivity and specificity estimates for the diagnosis of rifampicin-resistant TB were excellent and consistent, across all subgroups, assay versions and specimen types. Thus, the GenoType assay has very high accuracy for rifampicin resistance. This is reflected in the high LR+ estimate and the very low LR- estimate, suggesting an excellent ability to both rule in and rule out rifampicin resistance.

The accuracy for isoniazid-resistant TB was variable, with sensitivity lower and more inconsistent than specificity. While specificity did not vary across subgroups, sensitivity was higher when only MTBDRplus studies were pooled; the highest accuracy for isoniazid resistance was obtained with the MTBDRplus assay, with the sensitivity improving to nearly $90 \%$.

A notable advantage of molecular tests is their rapid turnaround time, which may have implications for patient management and transmission of drug-resistant TB. The turn-around time for the MTBDR assays ranged from $6 \mathrm{~h}$ to 2 days, substantially faster than conventional DST. The latter was reported from a high-volume laboratory in South Africa [17]. Another key advantage is the direct use of line probe assays on clinical specimens; this precludes the need to wait for cultures to grow.

\section{Strengths and limitations of the meta-analysis}

The meta-analysis had several strengths. First, a standard protocol was used for carrying out the systematic review [11], including a comprehensive search strategy. Moreover, two reviewers independently carried out various stages of the systematic review process, including article selection and data

TABLE 7 Pooled summary estimates for isoniazid resistance

\begin{tabular}{|c|c|c|c|c|c|}
\hline Subgroup & Pooled sensitivity & Pooled specificity & Pooled LR+ & Pooled LR- & Pooled DOR \\
\hline All isoniazid studies ${ }^{\#}$ & $84.3(76.6-89.8)$ & 99.5 (97.5-99.9) & $190.6(33.4-1086.3)$ & $0.16(0.10-0.24)$ & $1210.8(175.3-8361.5)$ \\
\hline Only MTBDRplus assays & $88.7(82.4-92.8)$ & $99.2(95.4-99.8)$ & $112.6(18.7-677.7)$ & $0.11(0.07-0.18)$ & 986.8 (133.6-7285.9) \\
\hline Only clinical specimens ${ }^{+}$ & 84.5 (72.1-92.0) & $99.2(96.4-99.8)$ & $110.1(22.3-542.3)$ & $0.15(0.08-0.29)$ & 706.6 (97.7-5110.8) \\
\hline
\end{tabular}

Data are presented as \% (confidence interval). MTBDRplus is manufactured by Hain LifeScience GmbH (Nehren, Germany). LR+: positive likelihood ratio; LR-: negative likelihood ratio; DOR: diagnostic odds ratio. ${ }^{*}$ : number of comparisons was $15 ; "$ : number of comparisons was 5 ; ${ }^{+}$: number of comparisons was 10 . 
extraction. Lastly, rigorous methods were used for data analysis, including bivariate random effects models, SROC analyses and methods for exploring heterogeneity.

The meta-analysis was limited by the relatively small number of available studies and the types of outcomes reported in the studies. Most studies only presented data on sensitivity and specificity. An obvious limitation is the lack of data on whether or not line probe assays have a clinical impact on patient management and treatment outcomes, and how much value they contribute beyond conventional tests. Data are also lacking on cost-effectiveness and feasibility in routine programme settings. Furthermore, there is little current evidence on how line probe assays may fit into existing diagnostic and treatment algorithms.

Despite using subgroup analysis, considerable heterogeneity remained unexplained in the isoniazid sensitivity results; further work is necessary to determine why isoniazid sensitivity values vary across settings. Geographic and genetic variations in the distribution of drug-resistant strains of $M$. tuberculosis might partially explain the present finding. For example, the prevalence of mutations in the inhA and kat $G$ genes seems to vary widely in different geographic locations [17]. Furthermore, there were inadequate data to stratify by smear status, as smear-negative patients are the group most likely to benefit from the use of molecular assays.

Finally, the present authors excluded studies published in languages other than English. This, combined with potential publication bias, may have resulted in an overly optimistic estimate of the accuracy of the GenoType MTBDR assays. Currently available statistical approaches for publication bias (e.g. funnel plots and regression tests) are not recommended for diagnostic meta-analysis [37], and it is therefore difficult to rule out potential publication bias in the meta-analysis. In addition, the rates of indeterminate MTBDR results ranged $1.4-19.2 \%$ across studies. Since these were often not included in the calculations for sensitivity and specificity, the reported accuracy estimates may be inflated to some degree.

\section{Conclusions}

GenoType MDTBR assays demonstrate excellent accuracy for rifampicin resistance, which is a proxy for MDR-TB. This suggests good utility as a rapid screening tool, especially in settings with high rates of MDR-TB or HIV (where appropriate infection control is a major concern). While specificity is excellent for isoniazid, sensitivity estimates are modest and highly variable. With the latest MTBDRplus version of the assay, sensitivity for isoniazid resistance improves to $\sim 90 \%$. This could be further improved in future generation assays once more data on mutations conferring isoniazid resistance becomes available. In addition, while some studies included smear-negative or nonrespiratory specimens, the results as presented did not allow for separate calculations of sensitivity and specificity estimates. Further studies are needed to compare the accuracy of the MTBDR assays in smear-positive versus smear-negative patients and pulmonary versus extrapulmonary cases.

An important issue that remains is the affordability of molecular assays and the associated laboratory infrastructure needs in resource-constrained settings. Commercial molecular tests, with prices typically higher than conventional tests, are popular in resource-rich settings. However, the most resourceconstrained countries tend to have the highest burden of MDR-TB cases and are least likely to benefit from expensive technologies because of high costs and lack of appropriate laboratory capacity. Given that line probe assays are nucleic acid amplification assays, the need for proper laboratory design, laboratory standard operating procedures and quality control to avoid cross-contamination is paramount [38].

Realising this need, several groups, including the World Health Organization, the Foundation for Innovative New Diagnostics, and the Stop Tuberculosis Partnership's New Diagnostics Working Group, have launched initiatives to improve global laboratory capacity and to make new diagnostics affordable and accessible [39, 40]. For example, the Foundation for Innovative New Diagnostics has negotiated reduced pricing for the public and nonprofit sectors in lowresource countries, resulting in a substantial reduction of the cost of the MTBDRplus assay relative to conventional liquid culture and drug-susceptibility testing [17]. In addition, field demonstration projects are ongoing to evaluate the feasibility, cost and impact of these assays in routine programme conditions [17, 40]. Together with meta-analysis data, these real-world field studies will provide the evidence necessary for policy making and clinical practice. In fact, in June 2008, the World Health Organization announced a new policy statement, endorsing the use of line probe assays for the rapid screening of patients at risk of multidrug-resistant tuberculosis [41]. The recommended use of line probe assays is currently limited to culture isolates and direct testing of smear-positive sputum specimens. Line probe assays are not recommended as a complete replacement for conventional culture and drugsusceptibility testing.

\section{ACKNOWLEDGEMENTS}

The authors would like to thank R. O'Brien (Foundation for Innovative New Diagnostics (FIND), Geneva, Switzerland), H. Albert (FIND) and D. Hain (Nehren, Germany) for providing lists of relevant studies. The authors are also grateful to $\mathrm{K}$. Weyer (World Health Organization, Geneva, Switzerland) for helpful input and feedback on the current manuscript.

\section{REFERENCES}

1 World Health Organization. Global Tuberculosis Control: Surveillance, Planning, Financing. Report No. WHO/ HTM/TB/2006.362. Geneva, World Health Organization, 2006.

2 Centers for Disease Control and Prevention. Emergence of Mycobacterium tuberculosis with extensive resistance to second-line drugs - worldwide, 2000-2004. MMWR Morb Mortal Wkly Rep 2006; 55: 301-305.

3 Migliori GB, Loddenkemper R, Blasi F, Raviglione MC. 125 years after Robert Koch's discovery of the tubercle bacillus: the new XDR-TB threat. Is "science" enough to tackle the epidemic? Eur Respir J 2007; 29: 423-427.

4 Raviglione MC, Smith IM. XDR tuberculosis-implications for global public health. N Engl J Med 2007; 356: 656-659.

5 Heifets LB, Cangelosi GA. Drug susceptibility testing of Mycobacterium tuberculosis: a neglected problem at 
the turn of the century. Int J Tuberc Lung Dis 1999; 3: 564-581.

6 Dinnes J, Deeks J, Kunst H, et al. A systematic review of rapid diagnostic tests for the detection of tuberculosis infection. Health Technol Assess 2007; 11: 1-196.

7 World Health Organization. The Global MDR-TB and XDR-TB Response Plan, 2007-2008. Report No. WHO/ HTM/TB/2007.387. Geneva, World Health Organization, 2007.

8 Rossau R, Traore H, De Beenhouwer H, et al. Evaluation of the INNO-LiPA Rif.TB assay, a reverse hybridization assay for the simultaneous detection of Mycobacterium tuberculosis complex and its resistance to rifampin. Antimicrob Agents Chemother 1997; 41: 2093-2098.

9 Morgan M, Kalantri S, Flores L, Pai M. A commercial line probe assay for the rapid detection of rifampicin resistance in Mycobacterium tuberculosis: a systematic review and meta-analysis. BMC Infect Dis 2005; 5: 62.

10 Hain Lifescience GmbH. GenoType MTBDRplus, version 1.0. Available from: www.hain-lifescience.com/pdf/ 304xx_pbl.pdf Date created May 1, 2008. Date last accessed June 27, 2008.

11 Pai M, McCulloch M, Enanoria W, Colford JM Jr. Systematic reviews of diagnostic test evaluations: What's behind the scenes? ACP J Club 2004; 141: A11-A13.

12 Gatsonis C, Paliwal P. Meta-analysis of diagnostic and screening test accuracy evaluations: methodologic primer. AJR Am J Roentgenol 2006; 187: 271-281.

13 Reitsma JB, Glas AS, Rutjes AW, Scholten RJ, Bossuyt PM, Zwinderman AH. Bivariate analysis of sensitivity and specificity produces informative summary measures in diagnostic reviews. J Clin Epidemiol 2005; 58: 982-990.

14 Deville WL, Buntinx F, Bouter LM, et al. Conducting systematic reviews of diagnostic studies: didactic guidelines. BMC Med Res Methodol 2002; 2: 9.

15 Weizenegger M. New improved DNA-isolation method for rapid and sensitive molecular detection of mutations associated with rifampin and isoniazid resistance in Mycobacterium tuberculosis. European Society for Mycobacteriology Conference, Athens, Greece, 2007. Available from www.esmycobacteriology.eu/abstract.htm.

16 Bang D, Bengard Andersen A, Thomsen VO. Rapid genotypic detection of rifampin- and isoniazid-resistant Mycobacterium tuberculosis directly in clinical specimens. J Clin Microbiol 2006; 44: 2605-2608.

17 Barnard M, Albert H, Coetzee G, O'Brien R, Bosman ME. Rapid molecular screening for multidrug-resistant tuberculosis in a high-volume public health laboratory in South Africa. Am J Respir Crit Care Med 2008; 177: 787-792.

18 Hillemann D, Rusch-Gerdes S, Richter E. Application of the Genotype MTBDR assay directly on sputum specimens. Int J Tuberc Lung Dis 2006; 10: 1057-1059.

19 Hillemann D, Rusch-Gerdes S, Richter E. Evaluation of the GenoType MTBDRplus assay for rifampin and isoniazid susceptibility testing of Mycobacterium tuberculosis strains and clinical specimens. J Clin Microbiol 2007; 45: 2635-2640.

20 Hillemann D, Weizenegger M, Kubica T, Richter E, Niemann S. Use of the genotype MTBDR assay for rapid detection of rifampin and isoniazid resistance in Mycobacterium tuberculosis complex isolates. J Clin Microbiol 2005; 43: 3699-3703.
21 Makinen J, Marttila HJ, Marjamaki M, Viljanen MK, Soini H. Comparison of two commercially available DNA line probe assays for detection of multidrug-resistant Mycobacterium tuberculosis. J Clin Microbiol 2006; 44: 350-352.

22 Miotto P, Piana F, Cirillo DM, Migliori GB. Genotype MTBDRplus: a further step toward rapid identification of drug-resistant Mycobacterium tuberculosis. J Clin Microbiol 2008; 46: 393-394.

23 Miotto P, Piana F, Penati V, Canducci F, Migliori GB, Cirillo DM. Use of genotype MTBDR assay for molecular detection of rifampin and isoniazid resistance in Mycobacterium tuberculosis clinical strains isolated in Italy. J Clin Microbiol 2006; 44: 2485-2491.

24 Somoskovi A, Dormandy J, Mitsani D, Rivenburg J, Salfinger M. Use of smear-positive samples to assess the PCR-based genotype MTBDR assay for rapid, direct detection of the Mycobacterium tuberculosis complex as well as its resistance to isoniazid and rifampin. J Clin Microbiol 2006; 44: 4459-4463.

25 Hadgu A. Discrepant analysis: a biased and an unscientific method for estimating test sensitivity and specificity. J Clin Epidemiol 1999; 52: 1231-1237.

26 Whiting P, Rutjes AW, Reitsma JB, Bossuyt PM, Kleijnen J. The development of QUADAS: a tool for the quality assessment of studies of diagnostic accuracy included in systematic reviews. BMC Med Res Methodol 2003; 3: 25.

27 Littenberg B, Moses LE. Estimating diagnostic accuracy from multiple conflicting reports: a new meta-analytic method. Med Decis Making 1993; 13: 313-321.

28 Deeks JJ. Systematic reviews in health care: systematic reviews of evaluations of diagnostic and screening tests. BMJ 2001; 323: 157-162.

29 Irwig L, Macaskill P, Glasziou P, Fahey M. Meta-analytic methods for diagnostic test accuracy. J Clin Epidemiol 1995; 48: 119-130.

30 Harbord R, Whiting P. Metandi: meta-analysis of diagnostic accuracy using hierarchical logistic regression. Stata J 2008 (in press).

31 Glas AS, Lijmer JG, Prins MH, Bonsel GJ, Bossuyt PM. The diagnostic odds ratio: a single indicator of test performance. J Clin Epidemiol 2003; 56: 1129-1135.

32 Lijmer JG, Bossuyt PM, Heisterkamp SH. Exploring sources of heterogeneity in systematic reviews of diagnostic tests. Stat Med 2002; 21: 1525-1537.

33 Higgins JP, Thompson SG. Quantifying heterogeneity in a meta-analysis. Stat Med 2002; 21: 1539-1558.

34 Rutjes AW, Reitsma JB, Di Nisio M, Smidt N, van Rijn JC, Bossuyt PM. Evidence of bias and variation in diagnostic accuracy studies. CMAJ 2006; 174: 469-476.

35 Pai M, O’Brien R. Tuberculosis diagnostics trials: do they lack methodological rigor? Expert Rev Mol Diagn 2006; 6: 509-514.

36 Lijmer JG, Mol BW, Heisterkamp S, et al. Empirical evidence of design-related bias in studies of diagnostic tests. JAMA 1999; 282: 1061-1066.

37 Tatsioni A, Zarin DA, Aronson N, et al. Challenges in systematic reviews of diagnostic technologies. Ann Intern Med 2005; 142: 1048-1055.

38 Borst A, Box AT, Fluit AC. False-positive results and contamination in nucleic acid amplification assays: 
suggestions for a prevent and destroy strategy. Eur J Clin Microbiol Infect Dis 2004; 23: 289-299.

39 Pai M, Ramsay A, O’Brien R. Evidence-based tuberculosis diagnosis. PLoS Med 2008; 5: e156.

40 Perkins MD, Cunningham J. Facing the crisis: improving the diagnosis of tuberculosis in the HIV era. J Infect Dis 2007; 196: Suppl. 1, S15-S27.
41 World Health Organization. Policy Statement. Molecular line probe assays for rapid screening of patients at risk of multidrug-resistant tuberculosis (MDR-TB). www.who.int/tb/features_archive/policy_statement.pdf Geneva, World Health Organization, 2008. Date last updated: June 27, 2008. Date last accessed: September 11, 2008. 